\title{
Buenos días: la clase de hoy es un juego ¿y porqué no?
}

\section{N. Montanes ${ }^{\text {a }}$, R. Balart ${ }^{\mathrm{a}}$, L. Sánchez-Nacher ${ }^{\mathrm{a}}$, L. Quiles-Carrillo ${ }^{\mathrm{a}}$ y V. Fombuena ${ }^{\mathrm{a}}$}

${ }^{a}$ Grupo de Innovación de Prácticas Académicas (GIPA), Universitat Politècnica de València, Plaza Ferrándiz y Carbonell s/n, Alcoy, Alicante (ESPAÑA).

\begin{abstract}
The authors have developed a game based on traditional question-andanswer challenge as an alternative method to master classes. An important part of the contents and topics of the subjects taught by the authors are developed using this new methodology. It is based on gamification, teamwork and collaborative learning combined with an adaptation of the Aronson puzzle technique. This game can be done in any type of environment or classroom, during the class time assigned to the subject and hardly requires additional material. On the other hand, with the development of the game, the Transversal Competences of the Universitat Politècnica de València "Comprehension and Integration", "Teamwork and Leadership", "Effective Communication" and "Planning and Time Management" are worked. Student satisfaction has been evaluated using this alternative teaching method presentin very positive results. Although the implementation of the method is recent, no barely records are available, it appears that the academic performance is increasing.
\end{abstract}

Keywords: master classes, gamification, collaborative learning, transversal competences.

\section{Resumen}

Los autores han desarrollado un juego basado en los tradicionales juegos de preguntas y respuestas como método alternativo a las clases magistrales. Una parte importante de los contenidos y temarios de las asignaturas impartidas por los autores son desarrollados mediante esta nueva metodología, que se basa en la gamificicación, en el trabajo en equipo y en el aprendizaje colaborativo combinado con una adapctación de la técnica puzzle de Aronson. Dicho juego se puede realizar en cualquier tipo de entorno o aula, en el tiempo de clase asiganado a la asignatura y apenas requiere de material adicional. Además con su aplicación se trabajan en clase al mismo tiempo las Competencias Tranversales de la Universitat Politècnica de València de "Comprensión e Integración", "Trabajo en Equipo y Liderazgo", "Comunicación Efectiva" y "Planificación y Gestión del Tiempo". Se ha evaluado la satisfacción del alumnado con respecto a este método alternativo de docencia con resultados muy positivos. Del mismo modo, aunque la implantación del método es reciente, no disponiendose de apenas registros, parece que se está logrando incrementar el rendimiento académico. 
Palabras clave: clase magistral, gamificación, aprendizaje colaborativo, competencias transversales.

\section{Introducción}

Durante la realización de diversos cursos formativos, varios compañeros de departamento entramos en contacto con nuevas e innovadoras prácticas pedagógicas. Consideramos oportuno intentar emplear estas nuevas prácticas pedagógicas en nuestras actividades docentes, pues mediante la aplicación de las mismas se ha comprobado que mejoran los resultados tanto académicos, como de aprendizaje de los alumnos. Diversos estudios demuestran que a las 24 horas de una sesión con experiencias prácticas, los alumnos retienen el $75 \%$ de los conocimientos adquiridos, mientras que este porcentaje va disminuyendo hasta un 50\% cuando se han adquirido mediante la discusión en grupo y hasta un 5\% si se trata de una lección magistral (SALINAS).

Tras un proceso de revisión de diversos documentos, publicaciones, etc. y de la captación de información, de un modo más exhaustivo, de las ventajas de implantar una docencia alejada de las clases magistrales en nuestras aulas, decidimos centrarnos en la gamificación, en el trabajo en equipo y el aprendizaje colaborativo, combinándolo con la técnica puzle de Aronson, para desarrollar una metodología docente propia. Además pretendíamos que, más allá del temario exacto con el que se aplicara, fuese algo semejante a los llamados "materiales a prueba de profesores" (BONAFE), de tal modo que pueda perdurar en el tiempo y de la que otros docentes puedan hacer uso.

Debemos puntualizar que la gamificación la entendemos como "el término utilizado para referirnos a la aplicación de mecánicas de juego en ámbitos que normalmente no son lúdicos" (DETERDING y col.) (LLAGOSTERA), pues existen otros autores que circunscriben su significado a la "aplicación de elementos conceptuales propios del diseño de videojuegos a entornos distintos del juego" (PRIETO MARTIN y col. 2014).

Por otro lado, al hablar del aprendizaje colaborativo, hacemos referencia al término acuñado por Vygotsky ya en 1978 (VIGOTSKY): "el aprendizaje colaborativo consiste en aprender de otros y con otros". Esto significa que, ante un proyecto común, cada individuo aporta su perspectiva individual, construyéndose un nuevo proyecto, lo que supone la necesidad de que se deba cooperar y colaborar en grupo.

En cuanto a la técnica puzle, es una técnica de aprendizaje cooperativo que permite que los alumnos compartan en grupo una gran cantidad de información (MARTINEZ y col.). Por ello es la utilizada para el desarrollo de una de las partes de la metodología docente que presentamos.

Consideramos que se hace imprescindible la sustitución de los sistemas clásicos de docencia, por nuevas prácticas educativas en las que el alumnado no sea un mero receptor de las informaciones y datos expuestos por el profesorado, sino un agente activo que intervenga en su propio proceso de enseñanza-aprendizaje. Hay que tratar de lograr que los conocimientos adquiridos a lo largo de la carrera perduren de tal forma que los alumnos no solo se conviertan, tras acabar sus estudios, en profesionales capaces, sino en sujetos

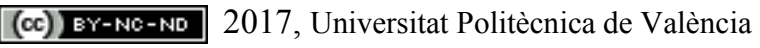


capaces de desarrollar e innovar. Lo que exponemos a continuación supone el intento de crear una metodología que resulte más eficaz y motivadora para los alumnos.

\section{Objetivos}

El objetivo principal es el desarrollo de un juego basado en los tradicionales juegos de preguntas y respuestas como método alternativo a las clases magistrales. Esta nueva metodología está basada en la gamificación, en el trabajo en equipo y en el aprendizaje colaborativo combinado con una adaptación de la técnica puzle de Aronson. Con todo ello lo que se pretende lograr es un incremento en el interés y motivación del alumnado, con el fin de maximizar los contenidos y/o conocimientos adquiridos por parte del mismo sobre los temas y materias tratadas en las aulas.

Por otro lado, la Universitat Politècnica de València (UPV) ha creado un proyecto con el objetivo de orientar sus prácticas docentes hacia el nuevo paradigma educativo que ve la educación superior como una formación basada en competencias. La UPV ha estipulado trece competencias transversales en su proyecto. El objetivo secundario de esta nueva metodología presentada es trabajar en las aulas cuatro de las trece competencias transversales de la UPV. Estas cuatro competencias tranversales de la UPV desarrolladas a través de esta nueva metodología son (UPV 2017):

- Comprensión e Integración: comprender quiere decir "percibir y tener una idea clara de lo que se dice, se hace o sucede o descubrir el sentido profundo de algo". Para demostrar que algo se ha comprendido, la persona identifica y recupera la información y la explica con sus propias palabras, interpretando e integrando las ideas desde su propia perspectiva.

- Trabajo en Equipo y Liderazgo: el trabajo en equipo implica crear y desarrollar un clima de confianza mutua entre los componentes que permita trabajar de forma responsable y cooperativa. El término más apropiado para describir esta situación es COMPARTIR: compartir conocimientos, compromiso y responsabilidad. Supone el reparto de tareas y roles y el respeto a las normas y reglas de juego establecidas por y para el grupo.

- Comunicación Efectiva: comunicarse efectivamente significa tener desarrollada la capacidad de transmitir conocimientos y expresar ideas y argumentos de manera clara, rigurosa y convincente, tanto de forma oral como escrita, utilizando los recursos apropiados adecuadamente y adaptándose a las circunstancias y al tipo de público.

- Planificación y Gestión del Tiempo: esta competencia implica ser capaz de organizar y distribuir correctamente el tiempo del que disponemos y distribuirlo en función de las actividades necesarias para alcanzar nuestros objetivos a corto, medio y largo plazo. 


\section{Desarrollo de la innovación}

Esta nueva metodología es aplicable en casi cualquier tipo de asignatura con mucha carga teórica, como alternativa a la clásica clase magistral. Además, se puede realizar en cualquier tipo de entorno o aula, en el tiempo de clase asignado a la asignatura y apenas requiere de material adicional.

Nuestra primera experiencia de aplicación fue en la asignatura de "Envase y Embalaje", con una asignación de 4.5 créditos en el tercer curso, segundo cuatrimestre, del Grado en Ingeniería en Diseño Industrial y Desarrollo de Productos. La media de alumnos por curso en esta asignatura se sitúa en 68. El tiempo de clase para el desarrollo de la parte teórica de la asignatura es de 2 horas semanales ( 15 semanas).

Las aulas en las que se imparte dicha asignatura de "Envase y Embalaje" presentan una distribución típica, con la zona del profesor en alto, con la la mesa, la pizarra y la pantalla del cañon-proyector y la zona de los alumnos más baja que la del profesor, con mesas y sillas formando filas. Esto no es un impedimento para la realización del juego, ya que tanto en la fase preparatoria del juego, como durante la fase de juego, las cuales se describen seguidamente, los alumnos se agrupan en distintas zonas del aula y si es necesario se le da la vuelta a determinadas mesas. El único material que deben traer los alumnos es lápiz y papel, y el tema impreso (en caso de no disponer de ordenadores o tablets en el aula; nuestro caso) para trabajarlo en clase. Los alumnos no han de preparar nada previamente a la sesión. Una vez terminada la sesión de juego, los alumnos habrán adquirido los concimientos trabajados, pero de una manera más agradable que la tradicional clase magistral.

En el presente curso académico estamos implantando esta metodología en otras varias asginaturas del área de Ciencia de los Materiales, esperando obtener resultados plenamente satisfactorios para todas las partes implicadas en la docencia.

A continuación, se detalla y describe esta nueva metodología.

\subsection{Reglas del Juego}

\subsubsection{Componentes del Juego}

Podemos diferenciar:

- Temario: tema a trabajar de una longitud media de 30 folios.

- Batería de preguntas: 30 o 35 preguntas tipo test por grupo, con 4 respuestas posibles, relacionadas al temario.

- Grupos: al menos dos grupos de un mínimo de 4 y un máximo de 6 alumnos por grupo.

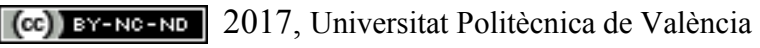


- Dado: sirve cualquier dado convencional que posea como mínimo el mismo número de caras que grupos van a jugar. No obstante, es conveniente que sea de proporciones especiales, es decir, lo suficientemente grande como para que todos los grupos lo puedan ver al mismo tiempo (por ejemplo, un cubo de $\pm 20 \mathrm{~cm}$ de lado).

\subsubsection{Bases del Juego}

El objetivo del juego radica en obtener el mayor número de puntos posibles a fin de ganar el juego, para lo cual habrá que responder correctamente, de forma conjunta, el mayor número de preguntas posibles de aquellas que realicen el resto de grupos.

No obstante, dado que no se trata de un mero juego de entretenimiento, sino que se persigue un objetivo pedagógico, se hace necesario un aliciente motivacional que impulse el deseo de ganar el juego, más allá de la mera satisfacción. Por ello, el ganar el juego supone la consecución de 0,4 puntos otorgados por el profesor a todos los miembros del grupo ganador, puntos que se sumaran a la nota obtenida en un próximo examen.

\subsection{Desarrollo del Juego}

En el desarrollo del juego podemos diferenciar dos fases: la fase preparatoria y la fase de juego.

\subsubsection{Fase Preparatoria}

Consiste en la preparación de los elementos necesarios para la realización del juego.

En esta fase:

- Los alumnos se dividen en grupos de 4 a 6 miembros cada uno, entre los que se nombrará un portavoz, un tira-dado y un cronometrador.

- Los grupos se numeran (grupo 1, grupo 2, etc.).

- El profesor proporciona al alumnado el tema a trabajar de una longitud media de 30 folios y los componentes del grupo se lo dividen, a fin de trabajar las partes de modo individual.

- Durante los siguientes 15 a 20 minutos, los alumnos deben trabajar de modo individual aquella parte del temario que les ha sido asignada, intentando comprender y asimilar los contenidos fundamentales.

- Una vez transcurrido el primer tiempo, los componentes del grupo deberán poner en común, uno a uno, los conocimientos comprendidos en su parte del temario, intentando que el resto de componentes del grupo tome conciencia de la materia, lo 
que no debería ocuparles más de unos 20-25 minutos (tiempo que de creerlo necesario se puede ampliar). De esta forma cada integrante del grupo asume el rol de profesor, tratando de transmitir los conocimientos que adquirido al trabajar, asimilar y comprender su parte del temario. Transcurrido este segundo tiempo cada integrante del grupo será conocedor del $100 \%$ del tema proporcionado por el profesor.

- Finalmente, los componentes de cada grupo, preferentemente de modo individual $\mathrm{y}$, de ser posible y quedarles tiempo, poniéndolo posteriormente en común, realizaran una batería 5-6 preguntas cada uno, en base a la parte del tema que han trabajado. Esta última etapa de la fase preparatoria no deberá durar más de 20 minutos.

Durante todas estas etapas el profesor debe estar para resolver aquellas dudas o problemas que puedan surgirles a los alumnos, así como para ayudarles a gestionar los tiempos. En base a la dificultad del temario y del alumnado con el que se trabaje, esta fase no debería superar la mitad del tiempo total de clase, en nuestro caso 1 hora.

\subsubsection{Fase de Juego}

La fase de juego comienza con la elección del grupo que iniciará el juego. Todos los grupos tiraran una vez el dado y el que saque un cinco será el que comience el juego. En caso de que más de un grupo saque dicho número, repetirían la tirada con el fin de desempatar.

Se inicia el juego y el tira-dado del grupo que lo hace tira el dado. El número que sale corresponderá al grupo que deberá realizarles la pregunta, el cual escogerá de su batería de preguntas aquella que considere conveniente y su portavoz se la leerá. El grupo que es preguntado dispondrá de 15 segundos para que su portavoz dé la respuesta, tiempo que controlará el cronometrador del equipo que les pregunta.

En caso de que acierten la pregunta que se les ha realizado, el grupo ganará un punto y volverá a tirar repitiéndose el proceso. En caso de que fallen la pregunta, el turno pasará al grupo colocado a su derecha, el cual realizará el mismo proceso.

\section{Resultados}

De la discusión y debate con otros docentes, así como con parte de nuestros alumnos en referencia a los cambios introducidos, se extrae la conclusión de una mejora en el interés y en los resultados académicos obtenidos, así como una mayor motivación por parte del alumnado.

Las clases son de asistencia obligatoria, por lo que el faltar a las mismas no es una posibilidad. Pero mediante la observación hemos comprobado que hay un mayor interés del alumnado por la concurrencia al aula desde la aplicación de esta nueva metodología, dado que llegan con más antelación a la necesaria y no manifiestan un denotado afán por irse cuando finaliza la clase.

2017, Universitat Politècnica de València 
No obstante, a fin de testear esta nueva metodología de un modo algo más empírico y menos subjetivo que la mera observación, realizamos un pase de encuestas en la asignatura anteriormente mencionada (Envase y Embalaje), mediante las cuales intentamos confirmar nuestras consideraciones.

Efectuamos una encuesta de cinco preguntas a los alumnos, solicitándoles que contestaran en base a tres parámetros: SI, NO y TAL VEZ.

La primera pregunta iba encaminada a averiguar sus preferencias en cuanto al tipo de clase, la segunda hacía referencia a la facilidad en la adquisición de los conocimientos, la tercera a los niveles motivacionales, la cuarta la conformamos como pregunta de control volviendo a referirnos al tipo de docencia y, por último, la quinta se refería al trabajo grupal. Estas eran las preguntas:

- Pregunta 1: ¿Preferirías que la asignatura se diera mediante una clase magistral?

- Pregunta 2: En cuanto a la adquisición de los conocimientos ¿Se facilita mediante el sistema de juego?

- Pregunta 3: ¿Te es fácil mantener el interés en el juego?

- Pregunta 4: ¿Te es más interesante la asistencia a una clase magistral?

- Pregunta 5: Si pudieras elegir ¿desearías trabajar de modo individual?

Finalizado el proceso de pase de encuestas y recopilación de respuestas, obtuvimos los resultados que se exponen en la Tabla 1:

Tabla 1. Resultados encuesta

\begin{tabular}{|l|c|c|c|}
\hline & SI & NO & TAL VEZ \\
\hline Pregunta 1 & $5 \%$ & $90 \%$ & $5 \%$ \\
\hline Pregunta 2 & $93 \%$ & $3.6 \%$ & $3.4 \%$ \\
\hline Pregunta 3 & $95 \%$ & $2 \%$ & $3 \%$ \\
\hline Pregunta 4 & $5 \%$ & $92.5 \%$ & $2.5 \%$ \\
\hline Pregunta 5 & $22 \%$ & $75 \%$ & $3 \%$ \\
\hline
\end{tabular}

Estos resultados nos llevan a unas conclusiones semejantes a las que llegamos mediante la mera observación de los comportamientos sociales del alumnado. Así pues, resulta evidente que la experiencia es positiva y comporta los esperados beneficios. Además, para el caso de la asignatura citada, Envase y Embalaje, pasamos de un $11.11 \%$ de alumnos suspendidos al final de la evaluación continua, a un $4.69 \%$ al siguiente año de aplicación de la nueva metodología descrita, lo que significa una mejora de más del doble del rendimiento académico. 


\section{Conclusiones}

Si bien ya lo hemos dicho en el apartado anterior, querríamos recalcar que la conclusión que se desprende tanto de la práctica docente realizada, como de los resultados obtenidos de la misma, supone afirmar que resulta altamente interesante el uso de este tipo de técnicas y metodologías, pues se incrementan los niveles de atención e interés del alumnado, lo que, a su vez reporta una mejora tanto en los resultados académicos, como en los propios del proceso de enseñanza-aprendizaje.

Así pues, consideramos que la modificación y sustitución de las clásicas clases de carácter magistral y sus consecuentes pruebas evaluativas, en las que la totalidad de la puntuación a obtener depende exclusivamente de la capacidad de memorización y repetición de conceptos y datos por parte del alumnado, por una docencia basada en un proceso enseñanza-aprendizaje en el que se integre e involucre a los alumnos, resulta en todo caso un gran acierto.

\section{Referencias}

BONAFE, J. M. (1993). Proyectos curriculares y práctica docente.

DETERDING, S., D. DIXON, R. KHALED and L. NACKE (2011). "From game design elements to gamefulness: defining "gamification"". Proceedings of the 15th International Academic MindTrek Conference: Envisioning Future Media Environments, Tampere, Finland, ACM.

LlAGOSTERA, E. (2012). "On gamification and persuasion." Proceedings of the SBGames, Rio de Janeiro, Brazil: 2-4.

MARTINEZ, J. and F. GOMEZ (2010). "La técnica puzzle de Aronson: descripción y desarrollo." Arnaiz, P.; Hurtado, Ma. D. y Soto, FJ (Coords.) 25.

PRIETO MARTIN, A., D. DIAZ MARTIN, J. MONSERRAT SANZ and E. REYES MARTIN (2014). "Experiencias de aplicación de estrategias de gamificación a entornos de aprendizaje universitario." ReVision 7(2).

SALINAS, J. (1995). Organización escolar y redes: Los nuevos escenarios del aprendizaje. "Cabero, J.; Martínez, F. (Coord.): Los nuevos canales en la enseñanza.", Centro de Estudios Ramón Areces S.A.: -.

UPV, U. P. d. V. (2017). "Competencias Transversales UPV." from http://www.upv.es/contenidos/COMPTRAN/, [Consulta: Marzo 2017].

VIGOTSKY, L. (2001). "Psicología pedagógica. Un curso breve." Argentina. Editorial Aique.: 67-468.

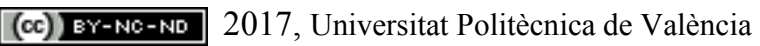

